\title{
Zum Stand der Paläolithforschung in der Schweiz
}

\begin{abstract}
:
Research on the Palaeolithic started in Switzerland during the 19th century in the overall framework of cave studies. Two different industries were soon recognized: middlepalaeolithic flake tools usually together with the bones of cave bear, and a late palaeolithic blade industry assoçiated with bone and antler tools, sculptures and line drawings. Recent research yielded complementary information on openair sites. A characteristic Mousterian tool-kit comes from a surface deposit at Pleigne JU, Löwenburg. New chronostratigraphic studies in the alpine Wildkirchli cave resulted in a relatively late date for its middle-palaeolithic layers. The late palaeolithic open-air and cave sites, which belong to an evolved Magdalenian tradition, seem to concentrate around the Bölling oscillation. Information on the final Palaeolithic and early Mesolithic is still too fragmentary for conclusions.
\end{abstract}

\section{Key Words:}

Cave Studies; Open-air Sites; Mousterian; Magdalenian; Chronostratigraphy.

Höhlensedimente mit eiszeitlichen Faunenresten und Belegen für menschliche Aktivitäten fanden in der Schweiz erstmals in der zweiten Hälfte des 19. Jahrhunderts Beachtung. Die Höhle von Cotencher, Gemeinde Rochefort NE, wurde 1858 entdeckt; eine erste Grabung erfolgte 1867, eingehende Untersuchungen 1916-1918 (DUBOIS u. STEHLIN 1932). Etwas später, 1874, setzten die ersten Sondierungen im «Kesslerloch», Gemeinde Thayngen SH, ein; nur die Grabungen von Jakob Heierli 1902-1903 bieten hier Ansätze einer Schichtbeobachtung (HEIERLI 1907).

Mit diesen beiden Jurastationen waren zugleich zwei Inventartypen grob umrissen, die in Höhlen ähnlicher Lage in der Folge wieder gefunden werden konnten: a) eine Abschlagindustrie zusammen mit Höhlenbärenknochen; b) eine jungpaläolithische Klingenindustrie mit begleitenden Rengeweihund Knochengeräten, gelegentlich auch Schmuck und Tierskulpturen bzw. Zeichnungen. Während sich die Kombination von Abschlaggeräten und Höhlenbärenknochen mit der Zeit auch in alpinen und voralpinen Höhlen einstellte (Wildkirchli, Wildenmannlisloch und Schnurenloch, vgl. Karte 1), fehlen aus diesem Milieu bis jetzt überzeugende jungpaläolithische Inventare.
Eine erste chronologische Einordnung der Höhlenfunde erfolgte mit Hilfe der Faunenreste im gleichen Schichtzusammenhang. Zu differenzierteren Ergebnissen führten seit 1953 die Sedimentanalyse (SCHMID 1958) und die Pollenanalyse (MÜLLER 1979).

Paläolithische Freilandstationen sind in der Schweiz gegenüber den Höhlen untervertreten. Die frühesten Nachweise stammen aus Ziegeleigruben und Steinbrüchen der Umgebung von Basel. In neuerer Zeit gesellen sich tiefe Baugruben hinzu, wobei aber Fundmeldungen leider die große Ausnahme bilden und flankierende geochronologische Untersuchungen ein dringendes Desiderat bleiben.

\section{Einzelfunde von Faustkeilen}

Potentiell günstig für die Entdeckung eines altsteinzeitlichen Lagerplatzes wäre zweifellos die Umgebung von Basel mit ihren Lößformationen, die nicht vom Eis des Würm-Glazials überfahren wurden. Einer der beiden Faustkeile der Schweiz kam 1975 denn auch hier, bei Pratteln BL, zum Vorschein (Karte 1). Leider erlaubt aber die Fundsituation keine eindeutige geochronologische Einordnung (HANTKE 1978, 301). Nicht anders steht es mit dem Faustkeil aus Schlieren bei Zürich (wYSS 1982).

\section{Höhlen und Freilandstationen mit Fundinventaren des Mittelpaläolithikums}

Das alpine Relief und die Gletschervorstöße während des Würm-Glazials setzten der Landnutzung durch Wildbeuterpopulationen im Gebiet der heutigen Schweiz Grenzen. Inter- und postglaziale Umlagerungen führten außerdem zu weitgehenden Informationsverlusten. Eine Übersicht über die vorhandenen Fundinventare soll die positiven wie die negativen Evidenzen beleuchten (Karte 1).

Margarita Primas, Prof. Dr., Abteilung für Ur- und Frühgeschichte des Historischen Seminars der Universität Zürich, Seilergraben 53, 8001 Zürich 
Mittelpaläolithische Industrien fanden sich in vier verschiedenen Lagetypen: in Jurahöhlen, in Höhlen der Alpen- und Voralpenzone, im Löß des Rheintals bei Basel und bei einer Silexlagerstätte im Jura. Die geochronologische Einordnung der alpinen Höhlen Wildenmannlisloch und Schnurenloch erfolgte mit Hilfe der Sedimentanalyse (SCHMID 1958) und der Pollenanalyse (MÜLLER 1979). Die Ergebnisse blieben aber mehrdeutig in dem Sinn, daß sich zwar Evidenzen für eine Ablagerung der fundführenden Sedimente in der Zeit vor dem Würm-Maximum ergaben, jedoch noch keine gesicherte Parallelisierung mit dem von der französischen Forschung an Höhlenstationen der Dordogne erarbeiteten Gliederungsschema (LAVILLE, RIGAUD, SACKETT 1980). Eine wohl wechselseitige Belegung jurassischer und alpiner Höhlen durch Menschen und Höhlenbären stellte zuletzt JÉQUIER (1975) heraus. Neue Untersuchungen erfolgten unter der Leitung von Elisabeth Schmid im Wildkirchli (SCHMID 1962). Hier lag die mittelpaläolithische Fundschicht über einem ca. $3 \mathrm{~m}$ mächtigen Schichtkomplex des frühen Würm-Glazials und konnte sedimentologisch mit dem Hengelo-Interstadial verknüpft werden (LE TENSORER 1986). Seit Jéquiers Arbeit steht fest, daß die überwiegend aus schlechtem Rohmaterial (Quarzit) hergestellten, durch Frosteinwirkung modifizierten Artefakte der alpinen Höhlen nur sehr bedingt morphologische Vergleiche erlauben.

Im Jura ergaben einstweilen nur die Höhle von Cotencher, Gemeinde Rochefort NE, und die Kastelhöhle, Gemeinde Himmelried SO, geochronologische und stratigraphische Evidenzen. Müller ordnete die obere der zwei Fundschichten von Cotencher in das Brørup-Interstadial ein, dies vor allem wegen auffallend kleinen Fichtenpollen, die er als picea omoricoïdes ansprach. In der Kastelhöhle war die Pollenerhaltung schlecht; die Sedimentanalyse zeigte, dass eine interstadiale Sinterbildung die mittelpaläolithische Fundschicht von den durch Lößeinwehungen und Frostabwitterung charakterisierten pleniglazialen Ablagerungen trennt. Über diesen folgen zwei jungpaläolithische Kulturschichten, womit sich - eine Ausnahme in der Schweiz - eine stratigraphische Abfolge ergibt (SCHWEIZER et al. 1959). Leider fehlen entsprechende Schichtbeobachtungen in einem wohl ähnlich gelagerten Fall, der Kohlerhöhle bei Brislach BE (LüDIN 1963).

Von den Freilandstationen wurde Münchenstein am besten überprüft (FURGER 1977). Hier fand sich eine Kombination kaltzeitlicher Faunenreste (Höhlenhyäne, wollhaariges Nashorn, Mammut, Ren, Pferd, Murmeltier u.a.m.) mit einer Abschlagindustrie, die allerdings nach Anzahl und formaler Variation noch wenig aussagefähig ist. Die Funde kamen zusammen mit Birkenholzkohle in bis zu $4 \mathrm{~m}$ mächtigen Lößtaschen auf Schotterformationen zum Vorschein.
Eine Sonderstellung nimmt die Freilandstation Löwenburg, Neumühlefeld III bei Pleigne JU, ein. Auf einem südexponierten Geländesporn brachte der Pflug immer wieder mittelpaläolithische Silexgeräte an die Oberfläche. Systematische Begehungen und zwei Sondierschnitte zeigten, daß kein ungestörter Lagerplatz vorliegt (JAGHER-MUNDWILER 1973-74). Während des Neolithikums war nämlich der in den anstehenden Malmkalk eingelagerte Silex bergmännisch abgebaut worden (SCHMID 1982). Man konnte daher das aufgesammelte Silexmaterial lediglich nach formalen Kriterien zeitlich ordnen. Nun findet sich darunter aber eine Geräteserie, deren Verhängung im Moustérien des südlichen Rhonetals möglich ist (LE TENSORER 1986). Zahlreiche Kernstücke in unterschiedlichen Abbaustadien machen klar, daß sich bei der Löwenburg ein paläolithischer Silexschlagplatz befand. Der Erhaltungszustand der retuschierten Geräte ist wesentlich besser als in den Höhlenstationen. Dazu kommt die Rohstoffselektion: selbst in Cotencher besteht nur ein kleiner Teil der Geräte aus Silex guter Qualität, wie er für die Löwenburg charakteristisch ist. Diese Fundstelle zeigt exemplarisch, daß Aussagen zur mittelpaläolithischen Besiedlung der Schweiz eine bessere Kenntnis offener Lager- und Schlagplätze erfordern, als wir sie heute besitzen.

\section{Höhlen und Freilandstationen mit Fundinventaren des späten Jungpaläolithikums}

Bislang fehlen im eiszeitlichen Spurenbild der Schweiz Nachweise der Aurignacien- und GravetteTechnokomplexe. Soweit diese Industrien in Kaltphasen fallen, während denen die Schweiz unter der alpinen Eisdecke lag, sind reale Zäsuren vorauszusetzen. Erst die Magdalénien-Industrie ist wieder vertreten, und zwar mit relativ jungen Inventaren, gemessen an der in Frankreich definierten Gesamterstreckung. Einstweilen bilden Jurahöhlen das dominante Milieu, doch muß dies als Folge des Höhlenforschungstrends zu Beginn unseres Jahrhunderts gesehen werden (vgl. Karte 2). Die 1983 entdeckte Seeuferstation Hauterive-Champréveyres NE wird sicherlich korrigierende Impulse zur Folge haben: hier kann erstmals gezeigt werden, wie die Uferzone eines Mittellandsees schon längst vor dem Mesolithikum in den Lebensraum einer Wildbeuterpopulation integriert wurde (SCHIFFERDECKER 1984). Die 14C-Daten machen ein etwas höheres Alter dieser Station im Vergleich zum Freilandlagerplatz Moosbühl, Gemeinde Moosseedorf BE wahrscheinlich: $12510 \pm 130$ BP; $12620 \pm 145$ BP; $12730 \pm 135$ BP für die obere der zwei Magdalénien-Schichten von Hauterive (LE TENSORER 1986; $\mathrm{BP}=$ nach archäologischen Konventionen Radiokarbonalter vor heute ohne Jahrringkalibrierung) und $12060 \pm 130$ 


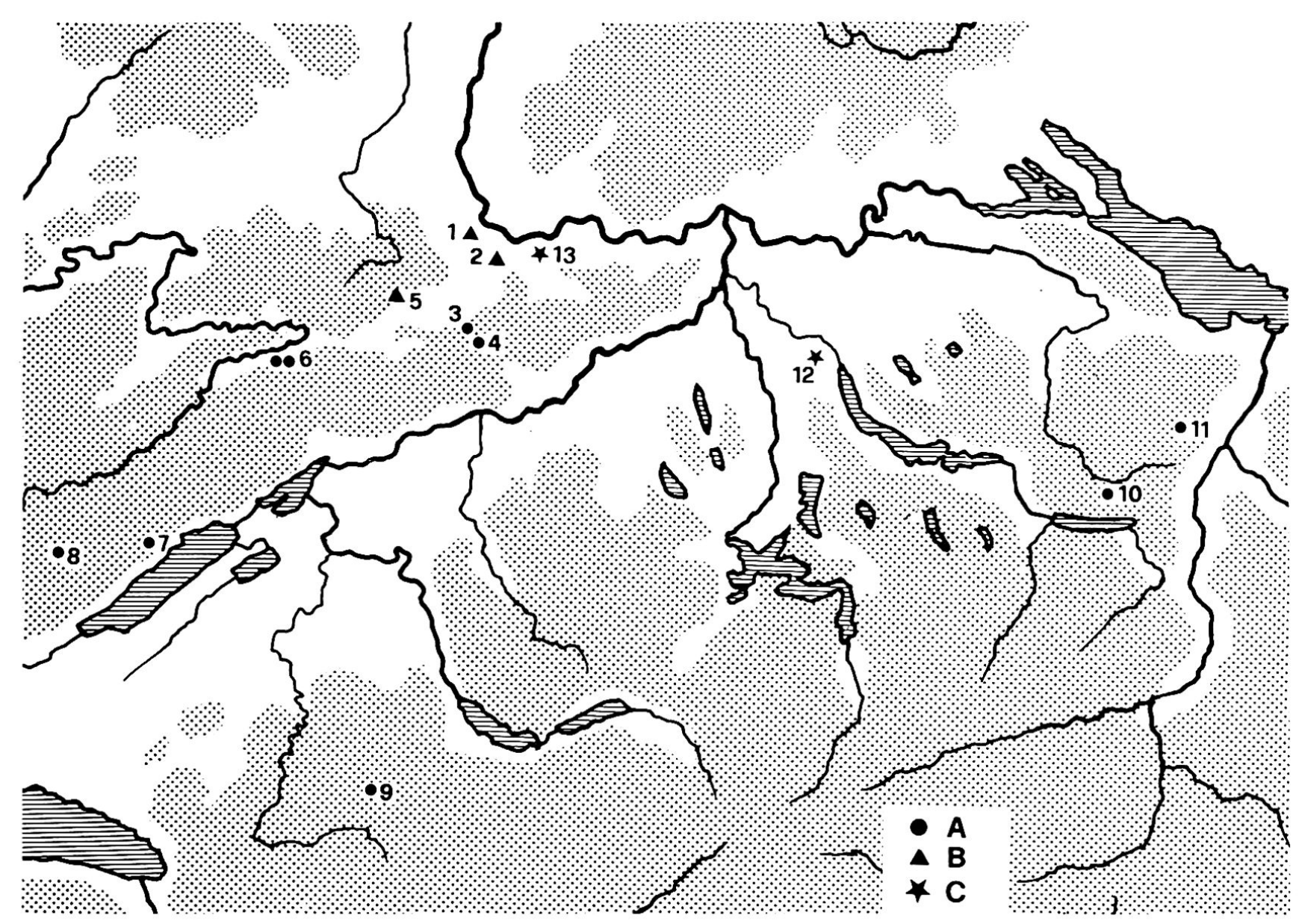

Karte 1: Fundstellen mittelpaläolithischer Abschlaggeräte in der Schweiz

Map 1: Distribution of middle-palaeolithic artifacts in Switzerland.
A Höhlen
B Freilandstationen
C Einzelfunde von Faustkeilen
A. Caves
B. Surface sites
C. Isolated hand-axes

(Nicht kartiert sind Fundstellen mit Höhlenbärenknochen, bei denen der Nachweis eines mittelpaläolithischen Gerätinventars aussteht. Zwei Faustkeile ohne Begleitinventar wurden in die Karte aufgenommen, obschon ihr Alter höher sein könnte.)
1. Allschwil BL, Ziegelei (Jb. SGUF 41, 1951, 41)
2. Münchenstein BL, Steinbruch (Furger 1977)
3. Pfeffingen BL, Schalbergfelsen (Vogt 1936)
4. Himmelried SO, Kastelhöhle (Schweizer et al. 1959)
5. Pleigne JU, Löwenburg (Jagher-Mundwiler 1973-74)
6. St-Brais JU, Höhlen 1 u. 2 (Jéquier 1975)
7. Rochefort NE, Cotencher (Dubois u. Stehlin 1932-33)
8. Couvet NE, Grotte des Plaints (Jéquier 1975)
9. Oberwil im Simmental BE, Schnurenloch (Andrist u. Flükiger 1964)
10. Alt St. Johann SG, Wildenmannlisloch (Jéquier 1975)
11. Schwende Al, Wildkirchli (Jéquier 1975)
12. Schlieren ZH (Wyss 1982)
13. Pratteln BL (d'Aujourd'hui 1977) 


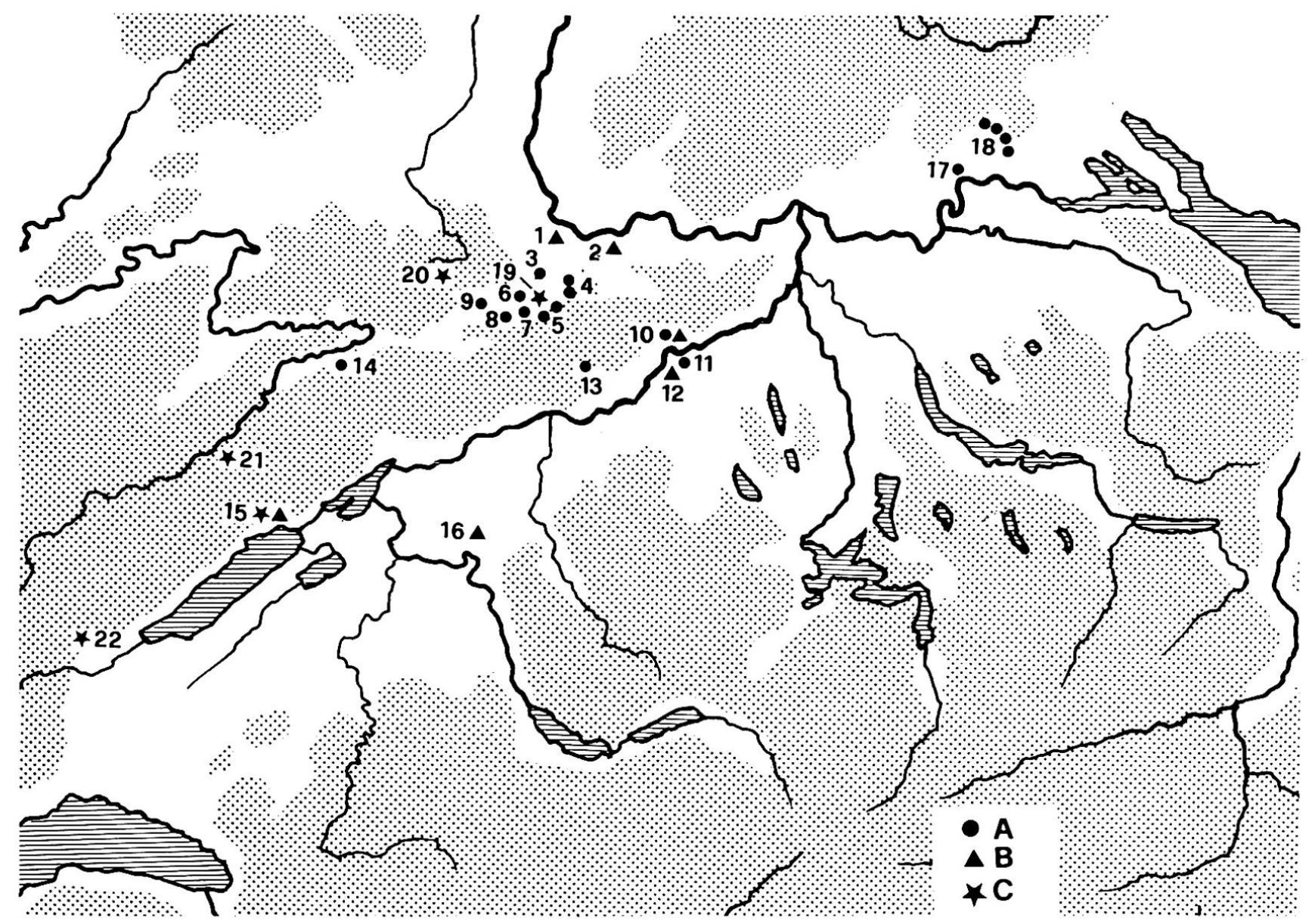

Karte 2: Fundstellen des Jung- und Endpaläolithikums in der Schweiz

Map 2: Distribution of late palaeolithic sites in Switzerland.
A Höhlen mit Magdalénien-Inventar
B Freilandstationen mit Magdalénien-Inventar
C Endpaläolithische Stationen
A. Caves with Magdalenian industry
B. Open-air sites with Magdalenian industry
C. Final palaeolithic sites.

(Unklare Fälle sind nicht aufgeführt; vgl. dazu Bandi 1947).

1. Basel-Gundeldingen, Bruderholz (Sedlmeier 1982)

2. Rheinfelden AG, Eremitage (Sedlmeier 1982)

3. Ettingen BL, Büttenloch (Sedlmeier 1982)

4. Arlesheim BL, Hollenberghöhle 3 und Birseck, Ermitage (Sedlmeier 1982)

5. Himmelried SO, Kastelhöhle und Heidenküche (Schweizer et al. 1959; Bandi 1947)

6. Nenzlingen BE, Brügglihöhle (Bandi et al. 1952-53)

7. Brislach BE, Kohlerhöhle (Lüdin 1963)

8. Büsserach SO, Thierstein (Bandi 1947)

9. Liesberg BE (Bandi 1947)

10. Winznau SO, Käsloch und Köpfli (Zürcher 1969)

11. Starrkirch-Wil SO, Mühleloch (Bandi 1947)

12. Olten SO, Sälihöhle-oben (Bandi 1947)

13. Oensingen SO, Rislisberghöhle (Barr 1977; Stampfli et al. 1983)

14. St-Brais JU (Bandi 1947)

15. Hauterive NE, Champréveyres (Schifferdecker 1984)

16. Moosseedorf BE, Moosbühl (Barr 1969-70; 1975; Schwab 1969-70)

17. Schaffhausen, Freudental (Bandi 1947; Bosinksi 1982)

18. Thayngen SH, Keßlerloch, Schweizersbild, Untere Bsetzi und Vorder Eichen (Bandi 1947; Joos 1982; Höneisen 1985)

19. Grellingen BE, Wachtfelsen (Lüdin 1960-61)

20. Roggenburg BE, Neumühle (Bandi 1967-68)

21. La Chaux-de-Fonds NE, Grotte du Bichon (Egloff 1980)

22. Baulmes VD, La Cure (Egloff 1979) 
BP; 11 180 \pm 120 BP; $11900 \pm 130$ BP für Moosbühl (BARR 1975). Auch die Ergebnisse der palynologischen Untersuchungen zeigten eine Altersdifferenz auf: Hauterive wird unmittelbar vor dem BöllingInterstadial eingeordnet (LE TENSORER 1986), während für Moosbühl der Übergang Bölling - ältere Dryas - vorgeschlagen wurde (BARR 1975). Es ergeben sich nun allerdings Probleme mit dem in anderen Gebieten erarbeiteten, höheren Zeitansatz für das Bölling. So weist beispielsweise BURGA (1987) für Graubünden 14C-Altersbestimmungen böllingzeitlicher Sedimente zwischen 13000 und 12000 BP nach, und im Mittelrheingebiet konnte der Magdalénien-Lagerplatz Feldkirchen-Gönnersdorf mit 14C-Daten um $12500 \mathrm{BP}$ datiert, geologisch und palynologisch aber gegen Ende des Bölling eingeordnet werden (BOSINSKI 1983). Noch größer ist die Diskrepanz zwischen der vegetationsgeschichtlichen und der durch ein 14C-Datum (11 860 $230 \mathrm{BP}$, STAMPFLI et al. 1983) nahegelegten Einordnung der 1971 entdeckten Rislisberghöhle, Gemeinde Oensingen SO. Die Fundschicht enthielt ein Magdalénien-Gerätinventar und Tierzeichnungen auf Knochen (BARR 1977). Nach den palynologischen Untersuchungen Wegmüllers (in STAMPFLI et al. 1983) dürfte zumindest das untere Niveau der Schicht noch vor der spätglazialen Wiederbewaldung (i.e. Bölling) unter kaltzeitlichen Bedingungen abgelagert worden sein.

Das Endpaläolithikum und die mit dem Übergang vom Spät- zum Postglazial verbundenen Veränderungen lassen sich am Fundbestand der Schweiz noch nicht adäquat diskutieren. Es fehlt einstweilen noch an 14C-datierten, chronostratigraphisch gut untersuchten Stationen, an denen sich der Faunenwechsel und die Silexgerätevolution zueinander in Beziehung setzen liessen. Eine im Alleröd abgelagerte Fundschicht, gefolgt von einem Hiatus während der jüngeren Dryaszeit, wurde unter dem Felsdach La Cure bei Baulmes VD festgestellt (EGLOFF 1979).

\section{Zusammenfassung:}

Die Paläolithforschung setzte in der Schweiz im 19. Jh. im weiteren Rahmen der Höhlenforschung ein. Zwei Inventartypen konnten unterschieden werden: mittelpaläolithische Abschlaggeräte in Assoziation mit Höhlenbärenknochen einerseits, eine jungpaläolithische Klingenindustrie mit Geweihgeräten und Kunstobjekten andererseits. Die neueren Forschungen legen mehr Gewicht auf Freilandstationen; in diesem Milieu wurde erstmals ein typisches Moustérien-Gerätinventar gefunden (Pleigne JU, Löwenburg). Chronostratigraphische Untersuchungen in der alpinen Höhle Wildkirchli präzisier- ten die relativ späte Zeitstellung dieser mittelpaläolithischen Station. Die jungpaläolithischen Freiland- und Höhlenlagerplätze ergaben fortgeschrittene bis späte Magdalénien-Inventare und scheinen sich zeitlich um das Bölling-Interstadial zu konzentrieren. Endpaläolithikum und Frühmesolithikum sind noch nicht ausreichend überblickbar.

\section{Bibliographie}

Jb. SGUF= Jahrbuch der Schweizerischen Gesellschaft für Ur- und Frühgeschichte, Basel.

$\mathrm{Jb} . \mathrm{BHMB}=$ Jahrbuch des Bernischen Historischen $\mathrm{Mu}$ seums, Bern.

ANDRIST D., FLÜKIGER W. u. ANDRIST A. (1964): Das Simmental zur Steinzeit. Acta Bernensia III, Bern.

D'AUJOURD'HUI R. (1977): Ein altpaläolithischer Faustkeil aus Pratteln BL. Festschrift f. Elisabeth Schmid zu ihrem 65. Geburtstag, Basel, S. 1-14.

BANDI H.G. (1947): Die Schweiz zur Rentierzeit. Huber, Frauenfeld.

BANDI H.G. (1967-68): Untersuchung eines Felsschutzdaches bei Neumühle, Gem. Pleigne, Kt. Bern. In: Jb. BHMB 47-48, S. 95-116.

BANDI H.G. et al. (1952-53): Die Brügglihöhle an der Kohlholzhalde bei Nenzlingen, Kt. Bern, eine neue Fundstelle des Spätmagdalénien im untern Birstal. In: Jb.BHMB 32-33, S. 45-76.

BARR J.H. (1969-70): Die Spätmagdalénien-Freilandstation Moosbühl. In: Jb.BHMB 49-50, S. 199-205.

BARR J.H. (1975): The Late Upper Palaeolithic Station of Moosbühl. A Geological Study and Dating of the Magdalenian Occupation. In: Mitt. Naturforsch. Ges. Bern NF. 32, S. 3-14.

BARR J.H. (1977): Die Rislisberghöhle, ein neuer Magdalénien-Fundplatz im Schweizer Jura. In: Archäologisches Korrespondenzblatt 7, S. 85-87.

BOSINSKI G. (1982): Die Kunst der Eiszeit in Deutschland und in der Schweiz. Kataloge vor- und frühgeschichtlicher Altertümer, Bonn.

BOSINSKI G. (1983): Eiszeitjäger im Neuwieder Becken. Landesamt f. Denkmalpflege Rheinland-Pfalz, Abt. Bodendenkmalpflege, Koblenz, 2. Aufl.

BURGA C.A. (1987): Das alpine Spät- und Postglazial in Graubünden aufgrund geomorphologischer und pollenanalytischer Untersuchungen. In: Vierteljahresschrift Naturforsch. Ges. Zürich 132/1, S. 26-44.

DUBOIS A. u. STEHLIN H.G. (1932-33): La grotte de Cotencher, station moustérienne. In: Mém. Soc. Paléont. Suisse 52, S. 1-178; 53, S. 179-292.

EGLOFF M. (1979): La transition du tardiglaciaire au postglaciaire en Suisse. In: Colloques intern. CNRS. 271, La fin des temps glaciaires en Europe, Talence. S. 231-237.

EGLOFF M. (1980): De Cotencher à Auvernier: l'âge de la pierre dans le canton de Neuchâtel. In: Helvetia Archaeologica 11, 43-44, S. 101-116. 
FURGER A.R. (1977): Die mittelpaläolithische Station beim unteren Steinbruch von Münchenstein. Festschrift $f$. Elisabeth Schmid zu ihrem 65. Geburtstag, Basel. S. 58-72.

HANTKE R. (1978): Eiszeitalter 1. Ott, Thun

HEIERLI J. (1907): Das Kesslerloch bei Thayngen. Neue Denkschr. Schweiz. Naturforsch. Ges. 43.

HÖNEISEN M. (1985): Neue ergänzte Lochstäbe aus dem Kesslerloch bei Thayngen (Kt. Schaffhausen, Schweiz). In: Archäologisches Korrespondenzblatt 15, S. 411-416.

JAGHER-MUNDWILERE. u. N. (1973-74): Die mittelpaläolithische Freilandstation Löwenburg im Berner Jura, Vorbericht. In: Jb. BHMB 53-54, S. 7-33.

Jéquier J.P. (1975): Le Moustérien Alpin. Révision critique. Eburodunum II - Cahiers d'Archéologie Romande 2, Yverdon.

JOOS M. (1982): Die Kernbohrungen von 1980 im Vorplatzbereich des Keßlerlochs (Thayngen $\mathrm{SH}$ ). Archäologie der Schweiz 5, 2, S. 46-50.

LAVILLE H., RIGAUD J.P., SACKETT J. (1980): Rock Shelters of the Périgord. Geological Stratigraphy and Archaeological Succession. Academic Press, New York/London.

LE TENSORER J.M. (1986): Paläolithikum und Mesolithikum. In: Chronologie, archäologische Daten der Schweiz. Antiqua 15. Schweiz. Ges. Ur- u. Frühgeschichte, Basel.

LÜDIN C. (1960-61): Mesolithische Siedlungen im Birstal. In: Jb.SGUF 48, S. 11-27.

LÜDIN C. (1963): Die Silexartefekte aus dem Spätmagdalénien der Kohlerhöhle. In: Jb.SGUF 50, 1963, 33-42.

MÜLLERE. (1979): Pollenanalytische Untersuchungen an paläolithischen und mesolithischen Höhlensedimenten aus der Schweiz und dem Vorarlberg. Antiqua 7. Schweiz. Ges. Ur- u. Frühgeschichte, Basel.
SCHIFFERDECKER F. (1984): Hauterive, distr. de Neuchâtel, Champréveyres. In: Jb.SGUF 67, S. 172.

SCHMID E. (1958): Höhlenforschung und Sedimentanalyse. Ein Beitrag zur Datierung des Alpinen Paläolithikums. Schriften Institut f. Ur- u. Frühgeschichte d. Schweiz 13, Basel.

SCHMID E. (1962): Ebenalp, Wildkirchlihöhle. In: Jb. SGUF 49, S. 39-42.

SCHMID E. (1982): Der neolithische Silexbergbau bei der Löwenburg (Pleigne JU). Archäologie der Schweiz 5, 2, S. 51-54.

SCHWABH. (1969-70): Moosbühl, Rettungsgrabung 1960. In: Jb. BHMB 49-50, S. 189-197.

SCHWEIZER Th. et al. (1959): Die Kastelhöhle im Kaltbrunnental, Gemeinde Himmelried, Solothurn. In: Jb. f. Solothurnische Geschichte 32, S. 3-85.

SEDLMEIER J. (1982): Die Hollenberg-Höhle 3. Eine Magdalénien-Fundstelle bei Arlesheim, Kt. BL. Basler Beiträge $z$. Ur- u. Frühgeschichte 8, Derendingen-Solothurn.

STAMPFLIH.R. et al. (1983): Rislisberghöhle. Archäologie und Ökologie einer Fundstelle aus dem Spätmagdalénien bei Oensingen im Solothurner Jura I. In: Academica Helvetica 4, 1. Bern-Stuttgart.

Ur- und frühgeschichtliche Archäologie der Schweiz I (1968): Die Ältere und Mittlere Steinzeit. Schweiz. Ges. Ur- u. Frühgeschichte, Basel.

VOGT E. (1936): Die paläolithische Station in der Höhle am Schalbergfelsen. In: Denkschr. Schweiz. Naturforsch. Ges. 71,3, S. 43-70.

WYSS R. (1982): Ein Faustkeil aus Schlieren bei Zürich. Jahresbericht Schweiz. Landesmuseum Zürich 91, S. 22-25.

ZÜRCHER A. (1969): Die spätjungpaläolithische Freilandstation Winznau-Köpfli. In: Jb. f. Solothurnische Geschichte 42, S. $138-201$. 